\title{
Autoimmunity to bactericidal/ permeability-increasing protein in bronchiectasis exhibits a requirement for Pseudomonas aeruginosa IgG response
}

To the Editor:

In bronchiectasis, due to diseases other than cystic fibrosis, idiopathic, genetic and environmental factors alter the airway landscape and immune responses, rendering the patients susceptible to infection, with the Gram-negative bacterium (GNB) Pseudomonas aeruginosa as a major contributor to mortality $[1,2]$. The high prevalence of $P$. aeruginosa in bronchiectasis patients cannot be explained by a single genetic or environmental influence, and immunological permissiveness of bronchiectasis airways to this colonisation remains unexplained [2]. A similar predisposition to this pathogen is characteristic of cystic fibrosis patients, in whom a single genetic mutation (CFTR) shapes the abnormal lung environment.

In cystic fibrosis, where $P$. aeruginosa colonises the airways of up to $80 \%$ of patients, we and others have proposed that the inability of the innate immune system to combat $P$. aeruginosa infection is related, in part, to an autoimmune antibody response to bactericidal/permeability-increasing protein (BPI), a neutrophil antimicrobial protein [3]. Through high-affinity binding of lipopolysaccharides (LPS) on the bacterial outer envelope, BPI mediates extracellular bactericidal and LPS neutralising functions [4]. Autoantibodies to BPI were first reported in European cystic fibrosis cohorts and confirmed by us in a US cohort of adult cystic fibrosis patients $[5,6]$. Notably, autoreactivity to BPI was associated with diminished lung function while in vitro functional studies demonstrated that anti-BPI IgG inhibits its biological activities [7-9].

In this research letter, we ask two critical questions regarding the immunological interactions that shape the bronchiectatic airway permissiveness to P. aeruginosa infection. 1) Do bronchiectasis patients develop autoimmunity to BPI? 2) What is the relationship between autoreactivity to BPI and chronic infection by $P$. aeruginosa? To address these questions, autoantibodies to BPI in patient sera were measured by ELISA in two bronchiectasis cohorts from the USA: one from Dartmouth Hitchcock Medical Center (DHMC) in Lebanon, NH ( $\mathrm{n}=16)$, and the other from Oregon Health and Science University (OHSU) in Portland, OR $(\mathrm{n}=42)$. Immunoblotting of sera negative by ELISA yielded a low frequency of additional seropositivity $(\sim 10 \%)$. BPI autoreactivity was identified at nearly identical frequencies in the DHMC $(56 \%)$ and OHSU $(52 \%)$ cohorts (figure $1 \mathrm{a}$ and $\mathrm{b})$.

We and others have reported an association between autoreactivity to BPI and the presence of $P$. aeruginosa in sputum culture of cystic fibrosis patients $[5,6]$. Given these findings, we evaluated the relationship between anti-BPI autoimmunity and chronic $P$. aeruginosa infection in bronchiectasis by measuring anti- $P$. aeruginosa IgG titres in patient sera as a serological marker of chronic infection. Healthy control sera exhibited little or no reactivity against PA14 extract (figure 1b). Autoreactivity to BPI in the DHMC bronchiectasis cohort was strongly associated with the existence of an antibody response to $P$. aeruginosa $(\mathrm{n}=16, \mathrm{p}=0.003)$ (figure $1 \mathrm{c})$. This association was confirmed in an independent cohort of bronchiectasis patients (OHSU; $n=42, p=0.002$ ) (figure 1d). Each bronchiectasis cohort exhibited a dichotomised relationship between anti-BPI autoreactivity and the presence of anti-P. aeruginosa antibodies (figure $1 \mathrm{c}$ and $\mathrm{d}$ ). Together, these findings represent the first report of anti-BPI autoimmunity

@ERSpublications

Large subpopulations of patients with bronchiectasis due to diseases other than cystic fibrosis $(\sim 50 \%$ in two independent cohorts) develop autoantibodies to BPI, which arise specifically in the context of chronic Pseudomonas aeruginosa infection http://ow.ly/1LHZ30mtfXu

Cite this article as: Skopelja-Gardner S, Theprungsirikul J, Meagher RE, et al. Autoimmunity to bactericidal/permeability-increasing protein in bronchiectasis exhibits a requirement for Pseudomonas aeruginosa IgG response. Eur Respir J 2019; 53: 1801891 [https://doi.org/10.1183/13993003.01891-2018]. 

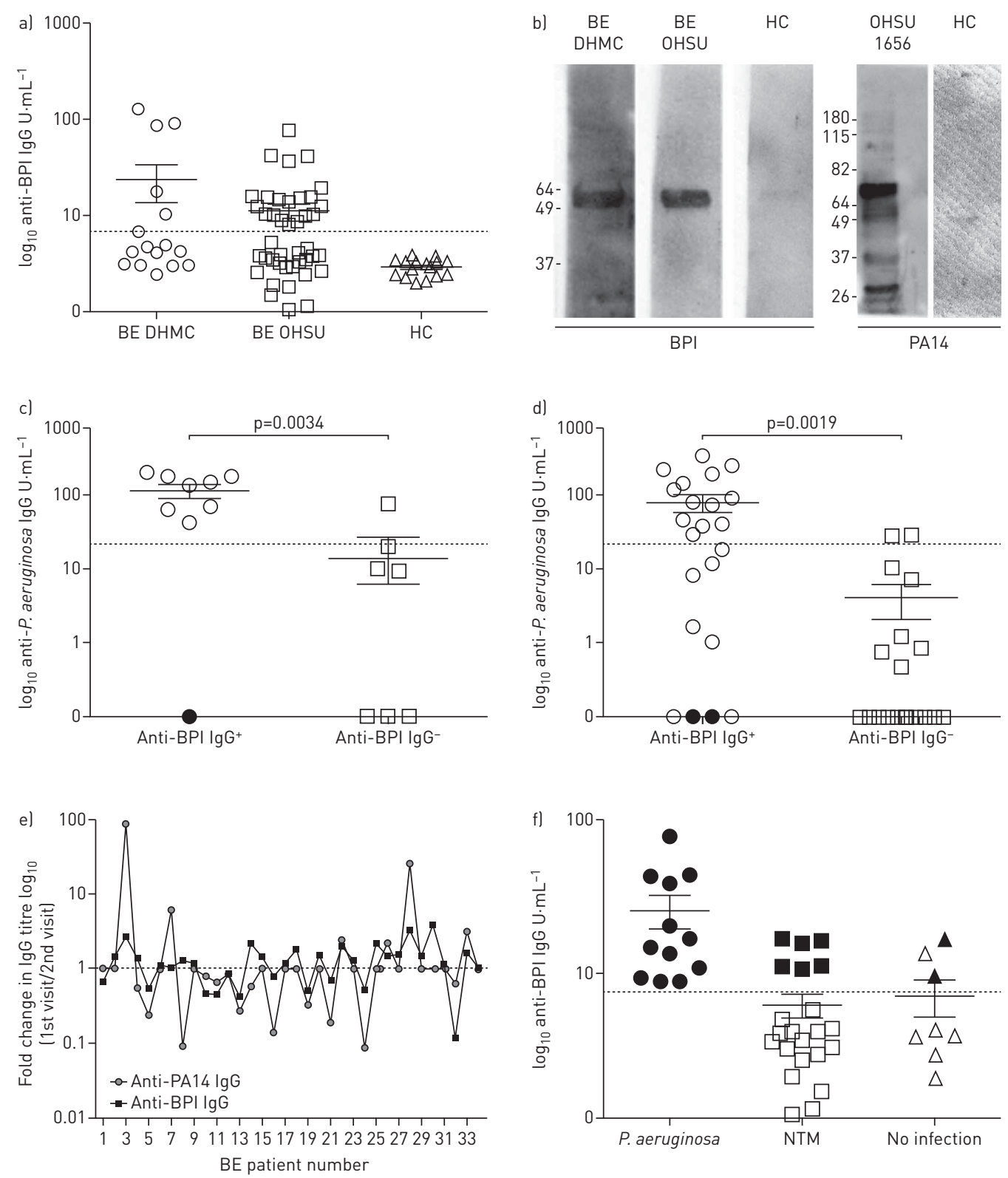

FIGURE 1 High anti-bactericidal/permeability-increasing protein (BPI) IgG titers in bronchiectasis (BE) patients associate with chronic Pseudomonas aeruginosa infection in a temporal and pathogen-specific manner. a) Anti-BPI IgG titers detected by ELISA in two BE cohorts: Dartmouth Hitchcock Medical Center (DHMC) ( $n=16,37.5 \%$ positive) and Oregon Health and Science University (OHSU) ( $=42,45.2 \%$ positive). Anti-BPI IgG positive samples $>6 \mathrm{U} \cdot \mathrm{mL}^{-1}$; positive cut-off determined as mean of healthy controls (HC) $+2 \mathrm{sD}$ $(n=16)$, represented by dashed line. b) Representative immunoblots of bronchiectasis serum reactivity to BPI $(5 \mu \mathrm{g})$ and $P$. aeruginosa PA14 lysate $(10 \mu \mathrm{g}) . \mathrm{c}$ and d) Anti-BPI lgG positivity, determined by ELISA and immunoblot, associates with antibody reactivity to $P$. aeruginosa (PA14 lysate) in both BE cohorts: C) BE DHMC and d) BE OHSU; reactivity to $P$. aeruginosa determined by ELISA (positive cut-off of $>22 \mathrm{U} \cdot \mathrm{mL}^{-1}$ represented by dashed line); filled symbols represent positive reactivity to $P$. aeruginosa by immunoblot. e) Fold change in anti-BPI and anti-P. aeruginosa IgG titers over two sequential visits in a retrospective longitudinal cohort of BE patients from $\mathrm{OHSU}$ ( $n=34 ; r=0.623 ; p=8.412 \times 10^{-5}$ as determined by Spearman correlation analysis). f) Anti-BPI IgG positivity in BE patients (OHSU) is associated with positive $P$. aeruginosa sputum culture. Positive sputum culture for nontuberculous mycobacterium (NTM) does not associate with anti-BPI IgG positivity in the absence of antibody response to $P$. aeruginosa. No infection: no current NTM infection. Filled symbols represent positive antibody reactivity to $P$. aeruginosa.

in bronchiectasis patients in the USA and indicate that autoreactivity to BPI develops specifically in the context of chronic $P$. aeruginosa infection, independently of a single genetic factor.

The relationship between the IgG antibody responses to $P$. aeruginosa and BPI was further examined through a retrospective longitudinal study of sera from 34 bronchiectasis patients. Serological analyses 
demonstrated that levels of antibodies targeting BPI and $P$. aeruginosa changed in the same direction over two consecutive visits; i.e. an increase in anti-P. aeruginosa IgG titres was accompanied by a positive fold change in anti-BPI autoantibody titres, while a reduction in anti- $P$. aeruginosa antibody levels tracked together with a negative fold change in anti-BPI IgG titres (figure 1e). Therefore, the autoimmune response to BPI follows the same temporal pattern as the humoral response to P. aeruginosa.

The specificity of this interaction with $P$. aeruginosa exposure and autoantibodies to BPI was also examined in relation to nontuberculous mycobacterium (NTM). Segregation of the OHSU bronchiectasis cohort by sputum culture yielded three patient populations: history of positive $P$. aeruginosa (with some overlapping NTM positivity within 6 months), NTM positive within 6 months, or no current infection. Serological analyses of each population demonstrated that anti-BPI IgG were absent in patients colonised with NTM or in those without an infection by sputum culture, unless accompanied by a positive anti- $P$. aeruginosa IgG titre (figure 1f). A positive sputum culture for $P$. aeruginosa was reported in only $55 \%$ of patients positive for anti- $P$. aeruginosa IgG, indicating that the serological analysis captures prior, as well as current, $P$. aeruginosa infection [10].

In this study, we report that anti-BPI autoreactivity in bronchiectasis is strongly associated with chronic $P$. aeruginosa infection, characterised by the presence of anti- $P$. aeruginosa antibodies. We observed this identical association in two bronchiectasis cohorts from New England and the Pacific Northwest. The synchronised changes in antibody reactivity to $P$. aeruginosa and BPI in a longitudinal cohort of bronchiectasis patients (figure 1e) further support the model that the breaking of tolerance to BPI is mediated through an association with chronic P. aeruginosa infection. The remarkable conservation of the linkage between autoreactivity to BPI and chronic P. aeruginosa infection in bronchiectasis is heightened by: 1) the heterogeneous genetic nature of bronchiectasis [11] and 2) identical associations in cystic fibrosis $[5,6]$. Together, these data argue against a human leukocyte antigen-dependent mechanism by which tolerance is broken, which is further bolstered by a similar relationship between BPI autoreactivity and immune response to $P$. aeruginosa in a bronchiectasis cohort from Japan [12]. Several potential mechanisms leading to the breach of tolerance to BPI in the context of $P$. aeruginosa infection warrant investigation: 1) molecular mimicry, 2) cross-activation of immune response due to LPS:BPI complexing and 3) cryptic epitope reveal due to differential BPI processing during inflammation. The latter model has been supported by our previous findings that $P$. aeruginosa-stimulated neutrophil extracellular trap formation leads to BPI cleavage and a possible reveal of neoepitope(s) [6].

The strength of the association between autoreactivity to BPI and chronic P. aeruginosa infection stands out in marked contrast to other autoantibodies against neutrophil azurophilic granule proteins (anti-neutrophil cytoplasmic antibodies (ANCA)), such as serine proteinase 3 and myeloperoxidase where no one infectious trigger has been defined [13]. In ANCA-associated vasculitis, while infectious stimuli have been implicated in the disease onset, their influence is only relevant in the context of genetic factors [14], unlike the $P$. aeruginosa-BPI interaction that argues against a genetic component of peptide restriction. Beyond the issue of immunopathogenesis of anti-BPI reactivity lie the functional implications of this autoreactivity. Three main functional roles of BPI have been proposed: 1) bactericidal, via LPS binding and permeabilisation of the GNB membrane; 2) inflammatory, via transport of GNB to dendritic cells; and 3) anti-inflammatory, via LPS neutralisation and down-modulation of monocyte response [15]. The temporal relationship between anti- $P$. aeruginosa and anti-BPI IgG titres suggests that autoimmune responses to BPI hinder its bactericidal and anti-inflammatory functions to facilitate colonisation/infection by $P$. aeruginosa. Thus, rather than viewing anti-BPI as a highly linked epiphenomenon of $P$. aeruginosa infection, these autoantibodies may play a causal role in the perpetuation of infection. In this model, strategies that eliminate anti-BPI reactivity may enhance clearance of $P$. aeruginosa, leading to improved airway function and clinical outcomes. Creating a model system in which the functional role of BPI and anti-BPI responses can be tested in vivo would seem to be a necessary first step in addressing this question.

Sladjana Skopelja-Gardner $\oplus^{1}$, Jomkuan Theprungsirikul ${ }^{1}$, Rachel E. Meagher ${ }^{1}$, Cathleen M. Beliveau ${ }^{1}$, Katherine E. Bradley ${ }^{1}$, Meade Avery ${ }^{1}$, Emily Henkle $\oplus^{2}$, Sarah Siegel $\odot^{2}$, Alex H. Gifford ${ }^{3,4}$, Kevin L. Winthrop ${ }^{2}$ and William F.C. Rigby ${ }^{1,5}$

${ }^{1}$ Dept of Microbiology and Immunology, Geisel School of Medicine at Dartmouth, Lebanon, NH, USA. ${ }^{2}$ Center for Infectious Disease Studies, OHSU-PSU School of Public Health, Portland, OR, USA. ${ }^{3}$ Division of Pulmonology, Dept of Medicine, Geisel School of Medicine at Dartmouth, Lebanon, NH, USA. ${ }^{4}$ The Dartmouth Institute for Health Policy and Clinical Practice, Lebanon, NH, USA. ${ }^{5}$ Division of Rheumatology, Dept of Medicine, Geisel School of Medicine at Dartmouth, Lebanon, NH, USA.

Correspondence: William F.C. Rigby, Division of Rheumatology, One Medical Center Drive, Lebanon, NH 03756, USA. E-mail: william.f.c.rigby@dartmouth.edu

Received: Oct 042018 | Accepted after revision: Oct 192018 
Acknowledgements: We thank Brent Berwin (Geisel School of Medicine at Dartmouth, Hanover, NH, USA) for providing the bacterial strain. We thank Ashley Switzer and Ashley Putz (Dartmouth Hitchcock Medical Center, Lebanon, $\mathrm{NH}$, USA) for procuring and handling DHMC bronchiectasis cohort samples.

Conflict of interest: None declared.

Support statement: This work was supported by the Clinical Research Scholars Program Award (GIFFOR17Y5).

\section{References}

1 Valderrey AD, Pozuelo MJ, Jimenez PA, et al. Chronic colonization by Pseudomonas aeruginosa of patients with obstructive lung diseases: cystic fibrosis, bronchiectasis, and chronic obstructive pulmonary disease. Diagn Microbiol Infect Dis 2010; 68: 20-27.

2 Foweraker JE, Wat D. Microbiology of non-CF bronchiectasis. In: Floto RA, Haworth CS, eds. Bronchiectasis (ERS Monograph). Sheffield, European Respiratory Society, 2011; pp. 68-96.

3 Aichele D, Schnare M, Saake M, et al. Expression and antimicrobial function of bactericidal permeability-increasing protein in cystic fibrosis patients. Infect Immun 2006; 74: 4708-4714.

4 Weiss J, Kao L, Victor M, et al. Oxygen-independent intracellular and oxygen-dependent extracellular killing of Escherichia coli S15 by human polymorphonuclear leukocytes. J Clin Invest 1985; 76: 206-212.

5 Lindberg $\mathrm{U}$, Carlsson $\mathrm{M}$, Hellmark $\mathrm{T}$, et al. BPI-ANCA provides additional clinical information to anti-Pseudomonas serology: results from a cohort of 117 Swedish cystic fibrosis patients. J Immunol Res 2015; 2015: 947934.

6 Skopelja S, Hamilton BJ, Jones JD, et al. The role for neutrophil extracellular traps in cystic fibrosis autoimmunity. JCI Insight 2016; 1: e88912.

7 Sediva A, Bartunkova J, Bartosova J, et al. Antineutrophil cytoplasmic antibodies directed against bactericidal/ permeability-increasing protein detected in children with cystic fibrosis inhibit neutrophil-mediated killing of Pseudomonas aeruginosa. Microbes Infect 2003; 5: 27-30.

8 Schinke S, Fellermann K, Herlyn K, et al. Autoantibodies against the bactericidal/permeability-increasing protein from inflammatory bowel disease patients can impair the antibiotic activity of bactericidal/permeability-increasing protein. Inflamm Bowel Dis 2004; 10: 763-770.

9 Schultz H, Heintz $\mathrm{H}$, van Zandbergen G, et al. ANCA against the bactericidal/permeability increasing protein (BPI-ANCA) can compromise the antibiotic function of BPI in a Wegener's granulomatosis patient. Clin Exp Rheumatol 2003; 21: 763-766.

10 Mauch RM, Jensen PO, Moser C, et al. Mechanisms of humoral immune response against Pseudomonas aeruginosa biofilm infection in cystic fibrosis. J Cyst Fibros 2018; 17: 143-152.

11 O’Donnell AE. Bronchiectasis. Chest 2008; 134: 815-823.

12 Ohtami S, Kobayashi O, Ohtami H. Analysis of intractable factors in chronic airway infections: role of the autoimmunity induced by BPI-ANCA. J Infect Chemother 2001; 7: 228-238.

13 Glasner C, de Goffau MC, van Timmeren MM, et al. Genetic loci of Staphylococcus aureus associated with anti-neutrophil cytoplasmic autoantibody (ANCA)-associated vasculitides. Sci Rep 2017; 7: 12211.

14 Willcocks LC, Lyons PA, Rees AJ, et al. The contribution of genetic variation and infection to the pathogenesis of ANCA-associated systemic vasculitis. Arthritis Res Ther 2010; 12: 202.

15 Schultz H, Hume J, Zhang DS, et al. A novel role for the bactericidal/permeability increasing protein in interactions of gram-negative bacterial outer membrane blebs with dendritic cells. J Immunol 2007; 179: $2477-2484$ 